\title{
Estimating dust distances to Type Ia supernovae from colour excess time-evolution
}

\author{
M. Bulla, ${ }^{\star}$ A. Goobar, R. Amanullah, U. Feindt and R. Ferretti \\ Oskar Klein Centre, Department of Physics, Stockholm University, SE 10691 Stockholm, Sweden
}

Accepted 2017 August 31. Received 2017 August 31; in original form 2017 July 3.

\begin{abstract}
We present a new technique to infer dust locations towards reddened Type Ia supernovae and to help discriminate between an interstellar and a circumstellar origin for the observed extinction. Using Monte Carlo simulations, we show that the time-evolution of the light-curve shape and especially of the colour excess $E(B-V)$ places strong constraints on the distance between dust and the supernova. We apply our approach to two highly-reddened Type Ia supernovae for which dust distance estimates are available in the literature: SN 2006X and SN 2014J. For the former, we obtain a timevariable $E(B-V)$ and from this derive a distance of $27.5_{-4.9}^{+9.0}$ or $22.1_{-3.8}^{+6.0} \mathrm{pc}$ depending on whether dust properties typical of the Large Magellanic Cloud (LMC) or the Milky Way (MW) are used. For the latter, instead, we obtain a constant $E(B-V)$ consistent with dust at distances larger than $\sim 50$ and 38 pc for LMC- and MW-type dust, respectively. Values thus extracted are in excellent agreement with previous estimates for the two supernovae. Our findings suggest that dust responsible for the extinction towards these supernovae is likely to be located within interstellar clouds. We also discuss how other properties of reddened Type Ia supernovae - such as their peculiar extinction and polarization behaviour and the detection of variable, blue-shifted sodium features in some of these events - might be compatible with dust and gas at interstellar-scale distances.
\end{abstract}

Key words: dust, extinction - circumstellar matter - supernovae: general - supernovae: individual: SN 2006X - supernovae: individual: SN 2014J

\section{INTRODUCTION}

The use of Type Ia supernovae (SNe Ia) as distance indicators has led to the breakthrough discovery of the accelerated expansion of the Universe (Riess et al. 1998; Perlmutter et al. 1999). Almost twenty years later, our ability to perform precision cosmology with $\mathrm{SNe}$ Ia is limited by various systematic uncertainties. Among these, one crucial uncertainty is given by our poor knowledge of what causes the colour-brightness relation found in SNe Ia. Redder SNe Ia are observed to be typically fainter than bluer ones, but whether this stems from intrinsic SN colour variations, dust reddening or a combination of the two is unclear (see e.g. Wang et al. 2009; Foley \& Kasen 2011).

The effect of dust on the observed reddening is typically described by the total-to-selective extinction ratio $R_{\mathrm{V}}=A_{\mathrm{V}} /\left(A_{\mathrm{B}}-A_{\mathrm{V}}\right) \equiv A_{\mathrm{V}} / E(B-V)$, where $A_{\mathrm{X}}$ denotes the extinction in a given band $X$. While $R_{\mathrm{V}} \sim 3$ is found for extinguished stars in our Galaxy (Fitzpatrick \& Massa 2007), significantly lower values are obtained for SNe Ia

* E-mail: mattia.bulla@fysik.su.se both from individual reddened events $(\sim 1.3-1.8$, Krisciunas et al. 2006; Elias-Rosa et al. 2006, 2008; Wang et al. 2008a; Folatelli et al. 2010; Amanullah et al. 2014, 2015) and larger samples $(\lesssim 2$, Tripp 1998; Astier et al. 2006; Nobili \& Goobar 2008; Kowalski et al. 2008; Burns et al. 2014 ). Similar low- $R_{\mathrm{V}}$ values are inferred from spectropolarimetric measurements and specifically from the observed correlation between extinction and wavelength of maximum polarization $\lambda_{\max }, R_{\mathrm{V}} \sim 5.5 \lambda_{\max }$ (Serkowski et al. 1975; Whittet \& van Breda 1978). While $\lambda_{\max } \sim 0.55 \mu \mathrm{m}$ and thus $R_{\mathrm{V}} \sim 3$ are typical values for Galactic stars, polarization spectra of some highly-reddened SNe Ia are characterized by $0.2 \lesssim \lambda_{\max } \lesssim 0.4 \mu \mathrm{m}$ and thus $1.1 \lesssim R_{\mathrm{V}} \lesssim 2.2$ (Patat et al. 2015; Zelaya et al. 2017).

One possible interpretation for the low- $R_{\mathrm{V}}$ towards some $\mathrm{SNe}$ Ia is that they arise from interstellar (IS) dust characterized by smaller grains than those in our Galaxy (see e.g. Gao et al. 2015; Nozawa 2016; Hoang 2017). In particular, Phillips et al. (2013) analysed spectra of 32 reddened SNe Ia and found a good correlation between extinction and strength of a diffuse interstellar band at $5780 \AA$, which they interpret as an evidence that the bulk of the ob- 
served reddening stems from IS clouds. The reason why the IS environment around SNe Ia appears to prefer small grains is still puzzling. However, Hoang (2017) recently suggested that a shift to smaller sizes might be the consequence of cloud-cloud collisions induced by the SN radiation pressure.

An alternative explanation for low- $R_{\mathrm{V}}$ values is that they arise from circumstellar (CS) dust (Wang 2005; Patat et al. 2006; Goobar 2008). In particular, Monte Carlo calculations by Goobar (2008) showed that the wavelengthdependence of scattering in CS dust can yield $R_{\mathrm{V}}$ values in the range between $\sim 1.5$ and 2.5 , in good agreement with observations. This interpretation would also be consistent with the presence of CS gas inferred for some SNe Ia from the observation of variable, blue-shifted sodium and potassium spectral features (Patat et al. 2007; Blondin et al. 2009; Simon et al. 2009; Sternberg et al. 2011; Foley et al. 2012; Maguire et al. 2013; Graham et al. 2015; Ferretti et al. 2016, but see also Chugai 2008 for a different interpretation within the IS scenario).

An interesting approach to estimate the dust location towards $\mathrm{SNe}$ is through the detection of light scattered by dust. These so-called light echoes were detected for a handful of SNe Ia and interpreted as arising in IS clouds (Schmidt et al. 1994; Sparks et al. 1999; Cappellaro et al. 2001; Quinn et al. 2006; Wang et al. 2008b; Crotts \& Yourdon 2008; Drozdov et al. 2015; Crotts 2015; Yang et al. 2017). Light echo measurements, however, are usually challenging as they require high-resolution (typically space-based), latetime imaging observations to distinguish contributions of scattered from direct light.

Here we present a new technique to infer dust locations in reddened SNe Ia and help discriminate between an IS and a CS origin of extinction. This is based on the peculiar effect of dust on the observed light-curve shape (Amanullah $\&$ Goobar 2011) and particularly on the $E(B-V)$ evolution with time. Details of our approach and of its implementation in Monte Carlo simulations are first described in Section 2. In Section 3, we test our technique and estimate dust locations for two well-studied highly-reddened SNe Ia for which dust distance estimates are available in the literature. Finally, we compare our findings to previous studies in Section 4 and draw conclusions in Section 5.

\section{SIMULATIONS}

In this section, we present results of our simulations. After a brief description of our Monte Carlo code (Section 2.1), we study how photons scattered by dust into the direct line-ofsight (DLOS) affect SN light-curve properties and provide constraints on dust location (Section 2.2). In particular, we will first address the impact on the light-curve width (Section 2.2.1) and then on the colour excess as a function of time (Section 2.2.2).

\section{$2.1 \quad$ Models}

We simulate the propagation of $\mathrm{SN}$ radiation in a dusty medium using a Monte Carlo code similar to those described by Goobar (2008) and Amanullah \& Goobar $(2011)^{1}$. A number $N_{\mathrm{ph}}$ of Monte Carlo photons are created at the origin and launched into the computational domain assuming isotropic emission. Each photon travels unperturbed until it reaches the dust region inner boundary (see below), at which point a random number $\xi_{1} \in[0,1)$ is drawn and the optical depth calculated as $\tau=-\log \left(1-\xi_{1}\right)$. The physical path length travelled by the photon, $d s$, is then calculated from

$\tau=\int n\left(\sigma_{\mathrm{s}}+\sigma_{\mathrm{a}}\right) d s$,

where $n$ is the number density of dust particles, and $\sigma_{\mathrm{s}}$ and $\sigma_{\mathrm{a}}$ are the scattering and absorption cross sections, respectively. Upon reaching the interaction point, a photon is either absorbed or scattered depending on the dust albedo

$a=\frac{\sigma_{\mathrm{s}}}{\sigma_{\mathrm{s}}+\sigma_{\mathrm{a}}}$.

Specifically, a second random number $\xi_{2} \in[0,1)$ is drawn and an absorption event selected when $\xi_{2}>a$, while a scattering event when $\xi_{2} \leqslant a$. In the former case, the absorbed photon is removed from the simulation and it does not contribute to the extracted light curve (see below); in the latter case, a new direction is chosen by sampling a scattering angle $\Theta$ from the Henyey-Greenstein function,

$\cos \Theta=\frac{1}{2 g}\left[1+g^{2}-\left(\frac{1-g^{2}}{1-g+2 g \xi_{3}}\right)^{2}\right]$,

where $\xi_{3}$ is a third random number chosen in the $[0,1)$ interval and $g=\langle\cos \Theta\rangle$ (Henyey \& Greenstein 1941; Witt 1977). The photon is then moved to the next interaction point and this procedure repeated until the photon is either absorbed or leaves the dust region. In the latter case, the escaping photon is saved and assigned (i) a final direction and (ii) a time delay with respect to a non-interacting photon emitted along the DLOS, $\Delta t$, calculated as in Amanullah \& Goobar (2011).

Following Amanullah \& Goobar (2011), the dust region is modelled as a spherical shell with outer radius $r_{\text {out }}$ and inner radius $r_{\text {inn }}=0.95 r_{\text {out }}$. In this work, $z=r_{\text {inn }}$ will be taken as representative for the distance between the SN and the dust region. For small distances $(z \lesssim 0.1 \mathrm{pc})$, this shell can be thought as representative of a spherical CS shell; for larger distances $(z \gtrsim 1 \mathrm{pc})$, it provides a good approximation to an IS dust slab ${ }^{2}$ at distance $z$. Wavelength-dependent dust properties $\left(a, \sigma_{\mathrm{a}}\right.$ and $g$ ) used in this work correspond to LMC - and MW-type dust. These are taken from Weingartner \& Draine (2001) and Draine (2003), respectively, and are the same as in table 1 of Goobar (2008). The number density $n$ is assumed to be constant throughout the dust region, and its value set to give a desired colour excess $E(B-V)_{\text {DLOS }}$ along the DLOS (Goobar 2008). In the

1 The code used in Amanullah \& Goobar (2011) is available at https://github.com/rahmanamanullah/csdust .

${ }^{2}$ For a photon scattered by dust into the DLOS, the path travelled is longer - and thus the time delay larger - in a slab compared to a spherical-shell case. However, for $z \gtrsim 1 \mathrm{pc}$ and for scattered photons reaching the observer at epochs considered here ( $t \lesssim 70 \mathrm{~d}$ since explosion), this time difference can be neglected as it is typically much smaller than $\Delta t$. 


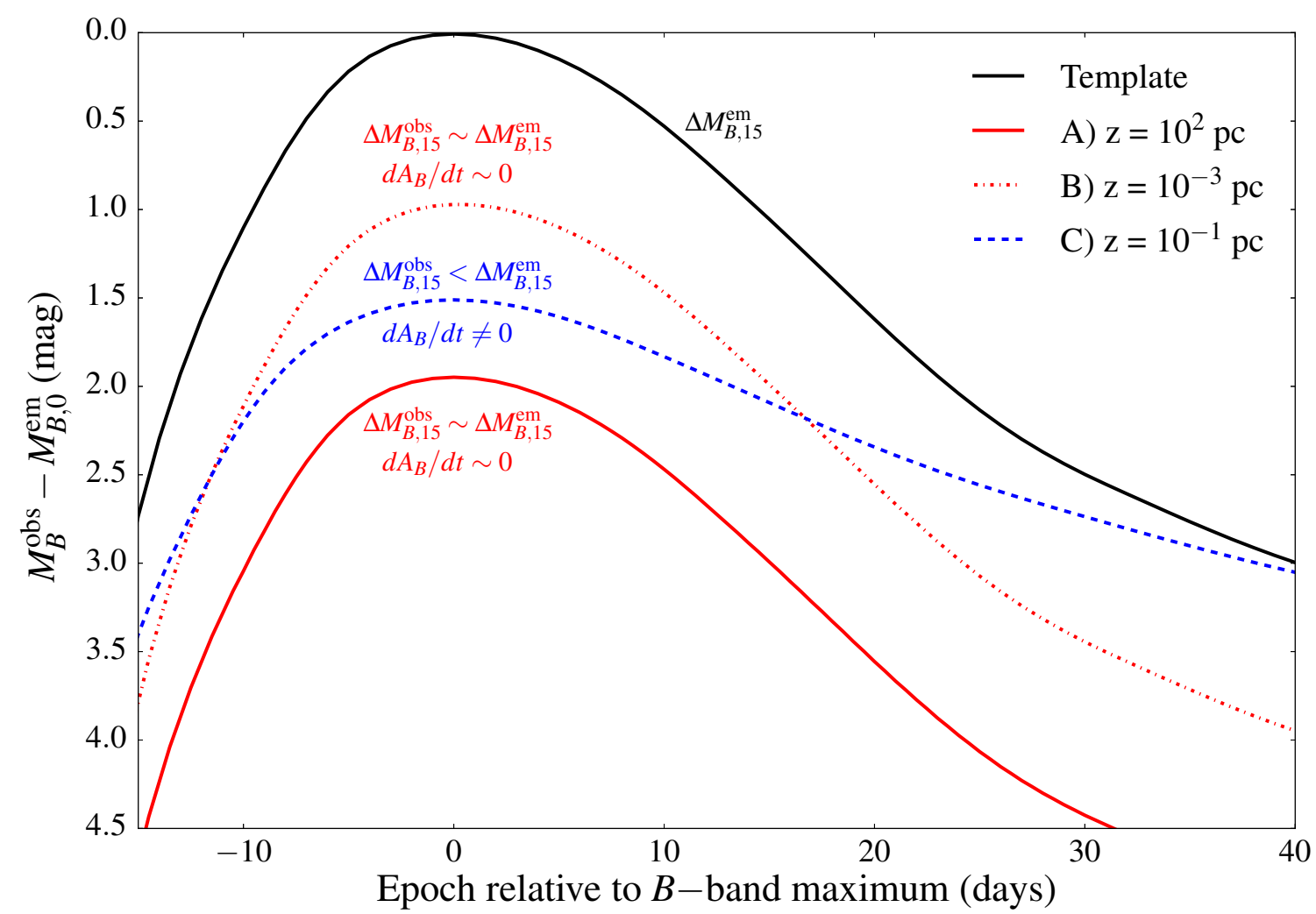

Figure 1. Effect of scattered photons on the $B$-band light-curve shape. A template $B$-band light curve, characterized by $\Delta M_{B, 15}^{\text {em }}$, is shown in black. Light curves reddened with LMC-type dust and $E(B-V)_{\mathrm{DLOS}}=0.5$ mag are shown for three different distances: $z=10^{2} \mathrm{pc}$ (solid red line), $z=10^{-3} \mathrm{pc}$ (dot-dashed red line), $z=10^{-1} \mathrm{pc}$ (dashed blue line).

case of a CS spherical shell, the assumed constant density implies that the total dust mass $M_{\text {dust }}$ increases with dust distance. Specifically, we find

$\frac{M_{\mathrm{dust}}}{M_{\odot}}=W\left[\frac{E(B-V)_{\mathrm{DLOS}}}{1 \mathrm{mag}}\right]\left[\frac{r_{\mathrm{inn}}}{0.01 \mathrm{pc}}\right]^{2}$,

where $W=9.5 \times 10^{-4}$ and $8.0 \times 10^{-4}$ for LMC- and MW-type dust, respectively. This equation is derived assuming a spherical shell for the dust region and thus the estimated mass is representative of a CS shell only (i.e. dust at small distances). In contrast, it does not apply to a cloud at large distances that should instead be thought as an IS dust slab with a given depth (see above).

Our Monte Carlo code is publicly available and can be found at https://github.com/mbulla/dust3d .

\subsection{Impact of scattered photons on the light curve}

Photons leaving the dust region and escaping to the observer are collected and used to construct multi-band light curves. As in Amanullah \& Goobar (2011), pristine SN Ia light curves are constructed from the Hsiao et al. (2007) spectral templates, which provides us with UBVRI fluxes from -20 to +85 d relative to $B$-band maximum. Using these, the SN emitted magnitude in a given band $X$ can be expressed as

$M_{X, \mathrm{t}}^{\mathrm{em}}=-2.5 \log f_{X}^{\mathrm{em}}(t)+C_{X}$, where $f_{X}^{\mathrm{em}}(t)$ is the flux emitted in the $X$-band at time $t$ since explosion, and $C_{X}$ some constant. At each given time $t$, the observed magnitude in the $X$-band is then calculated summing over all the escaping photons and taking into account their time delays $\Delta t$ (see Section 2.1):

$M_{X, \mathrm{t}}^{\mathrm{obs}}=-2.5 \log \frac{\sum_{i} f_{X}^{\mathrm{em}}\left(t-\Delta t^{i}\right)}{N_{\mathrm{ph}}}+C_{X}$.

Finally, the extinction $A_{X}(t)$ can be easily derived as

$A_{X, \mathrm{t}}=M_{X, \mathrm{t}}^{\mathrm{obs}}-M_{X, \mathrm{t}}^{\mathrm{em}}=-2.5 \log \frac{\sum_{i} f_{X}^{\mathrm{em}}\left(t-\Delta t^{i}\right)}{N_{\mathrm{ph}} f_{X}^{\mathrm{em}}(t)}$.

In the absence of dust extinction, for instance, all the emitted photons reaching the observer would have $\Delta t=0$, hence $M_{X, \mathrm{t}}^{\mathrm{obs}}=M_{X, \mathrm{t}}^{\mathrm{em}}$ and $A_{X, \mathrm{t}}=0$.

Following the work done by Amanullah \& Goobar (2011), here we investigate the impact of dust on the observed light curve. In particular, we choose to describe the light-curve shape by means of two parameters: (i) the widely-used decline rate in the $B$-band, $\Delta M_{B, 15}^{\mathrm{obs}}=M_{B, 15}^{\mathrm{obs}}-M_{B, 0}^{\mathrm{obs}}$, and (ii) the observed colour excess $E(B-V)=A_{B}-A_{V}$ relative to the pristine light curve. Fig. 1 shows $B$-band light curves for dust characterized by $E(B-V)_{\mathrm{DLOS}}=0.5 \mathrm{mag}$ and located at three different distances: 0.001, 0.1 and 100 pc. As already pointed out by Amanullah \& Goobar (2011), different dust locations translate into different light-curve shapes:

- At very large distances $(z=100 \mathrm{pc})$, the relative contribution of photons scattered into the DLOS and reaching 
the observer at epochs considered in this work $(t \lesssim 70 \mathrm{~d}$ since explosion) is negligible. Compared to the pristine light curve, the observed light curve is therefore fainter because of dust extinction but characterized by a similar shape, i.e. $\Delta M_{B, 15}^{\mathrm{obs}} \sim \Delta M_{B, 15}^{\mathrm{em}}$ and $A_{B}, A_{V}$ and thus $E(B-V)$ are constant with time.

- At very small distances $(z=0.001 \mathrm{pc})$, the relative contribution of photons scattered into the DLOS is significant and hence the observed light curve is brighter compared to the very-distant dust case described above. However, scattered photons arrive almost simultaneously with noninteracting photons $(\Delta t \sim 0)$ and thus the light-curve shape is again similar to the pristine one.

- At intermediate distances $(z=0.1 \mathrm{pc})$, the contribution of photons scattered into the DLOS is relatively large and their time delay of the order of days. Therefore, photons reaching the observer at a given time $t$ were emitted at different times and with different luminosities. This affects the observed light-curve shape significantly and translates into an observed broadening after maximum light $\left(\Delta M_{B, 15}^{\mathrm{obs}}<\Delta M_{B, 15}^{\mathrm{em}}\right)$. An effect of this light-curve broadening is to make $A_{B}, A_{V}$ and thus the colour excess $E(B-V)$ appear as time-variable.

As clearly seen at intermediate distances, we note that the extra-flux from scattered packets shapes the light curve not only decreasing $\Delta M_{B, 15}$ but also increasing the rise time to peak brightness. This effect was already shown and extensively discussed by Amanullah \& Goobar (2011, see section 6.3).

In the following, we investigate the two effects just discussed more closely and study how $\Delta M_{B, 15}^{\text {obs }}$ (Section 2.2.1) and $E(B-V)$ (Section 2.2.2) are affected by dust at different locations and with different $E(B-V)_{\mathrm{DLOS}}$ values. In particular, we focus on how these two parameters can be used to place constraints on the dust location towards $\mathrm{SNe}$ Ia.

\subsubsection{Light-curve broadening}

Fig. 2 shows the effect of light-curve broadening as a function of dust distance $z$ for different compositions and extinction values. Overall, the general behaviour in light-curve broadening is qualitatively similar between the different models: little broadening is observed for nearby $(z \lesssim 0.001 \mathrm{pc})$ and fairly distant $(z \gtrsim 100 \mathrm{pc})$ dust; larger broadening is observed for intermediate distances, with the exact extent depending on both dust composition and extinction.

First, we notice that the difference in dust composition is only marginally affecting the light-curve broadening, with LMC- and MW-type dust models predicting similar $\Delta M_{B, 15}^{\text {obs }}$ values at any dust distance. On the contrary, the dependence of light-curve broadening on the extinction is remarkable. As $E(B-V)_{\text {DLOS }}$ increases, the light-curve broadening becomes more significant and the dust distance at which the effect is maximum (corresponding to the curve minimum in Fig. 2) shifts towards larger values. While the former effect simply reflects the increasing contribution of scattered photons, the latter is a consequence of the increasingly larger distribution of time delays $\Delta t$ of photons reaching the observer.

The light-curve broadening for the $E(B-V)_{\mathrm{DLOS}}=0.1$

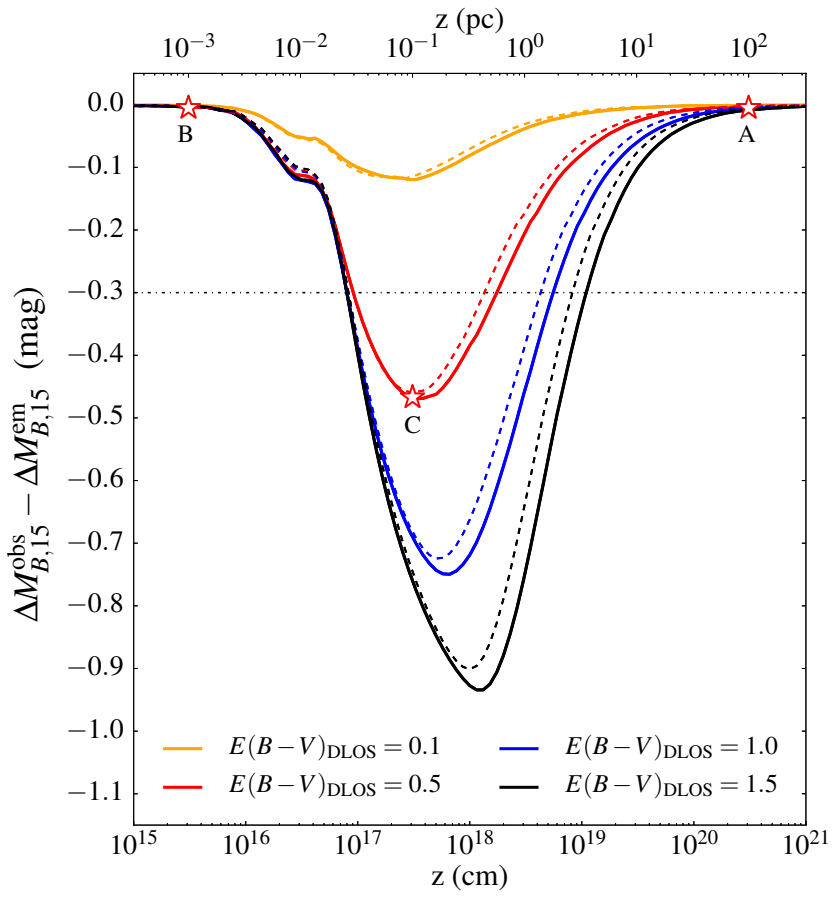

Figure 2. Light-curve broadening $\left(\Delta M_{B, 15}^{\mathrm{obs}}-\Delta M_{B, 15}^{\mathrm{em}}\right)$ as a function of dust distance $z$ for different $E(B-V)$ DLOS values. Solid lines refer to LMC-type dust, while dashed lines to MW-type dust. The horizontal dot-dashed line marks the light-curve broadening for a SN Ia with $\Delta M_{B, 15}^{\mathrm{em}}=1.1 \mathrm{mag}$ broadened to and observed as a slow-evolving SN Ia with $\Delta M_{B, 15}^{\text {obs }}=0.8 \mathrm{mag}$ (see text for explanation). For $E(B-V)_{\mathrm{DLOS}}=0.5 \mathrm{mag}$ (red lines), the three white stars highlight values corresponding to the A, B and C light curves of Fig. 1.

mag dust model is relatively moderate, with the change in $\Delta M_{B, 15}$ reaching a maximum of roughly 0.1 mag at $\sim 0.1$ pc. In contrast, larger values of $E(B-V)_{\text {DLOS leads }}$ to a broadening which is significant when dust is located at distances between 0.01 and a few pc. In this distance range, for instance, models with $E(B-V)_{\mathrm{DLOS}}=0.5 \mathrm{mag}$ are affected by changes in $\Delta M_{B, 15}$ that vary from roughly 0.15 to 0.5 mag. These numbers become even more extreme when going to larger extinction values, with 0.15 to 0.7 (0.9) mag changes in $\Delta M_{B, 15}$ for $E(B-V)_{\mathrm{DLOS}}=1.0$ (1.5) mag.

$\Delta M_{B, 15}^{\mathrm{em}}$ values for normal SNe Ia span a range between roughly 0.8 and $1.5 \mathrm{mag}$ (see e.g. Hillebrandt et al. 2013). For highly-extinguished SNe Ia, a range of intermediate distances $(\sim 0.01-1 \mathrm{pc})$ can thus be immediately ruled out as they would require extremely large value of $\Delta M_{B, 15}^{\mathrm{em}}$ (i.e. an extremely fast-declining pristine light curve). For instance, the well-studied SN 2006X (see also Section 3.1) was estimated to have an observed decline of $\Delta M_{B, 15}^{\text {obs }} \sim 1.1$ mag and a colour excess of $E(B-V) \sim 1.5 \mathrm{mag}$ (Wang et al. 2008a; Folatelli et al. 2010). In the case reddening along SN 2006X was due to dust located between 0.1 and $1 \mathrm{pc}$ from the SN, the pristine light curve would need to have unreasonably large decline rates for normal $\mathrm{SNe}$ Ia $\left(\Delta M_{B, 15}^{\mathrm{em}} \gtrsim 2 \mathrm{mag}\right)$. This argument would point - at least for highly-extinguished $\mathrm{SNe}$ Ia - towards dust located at either rather small $(z \lesssim$ $0.01 \mathrm{pc})$ or large $(z \gtrsim 1 \mathrm{pc})$ distances. We note, however, that nearby dust at $z \lesssim 0.01 \mathrm{pc}$ is in strong tension 


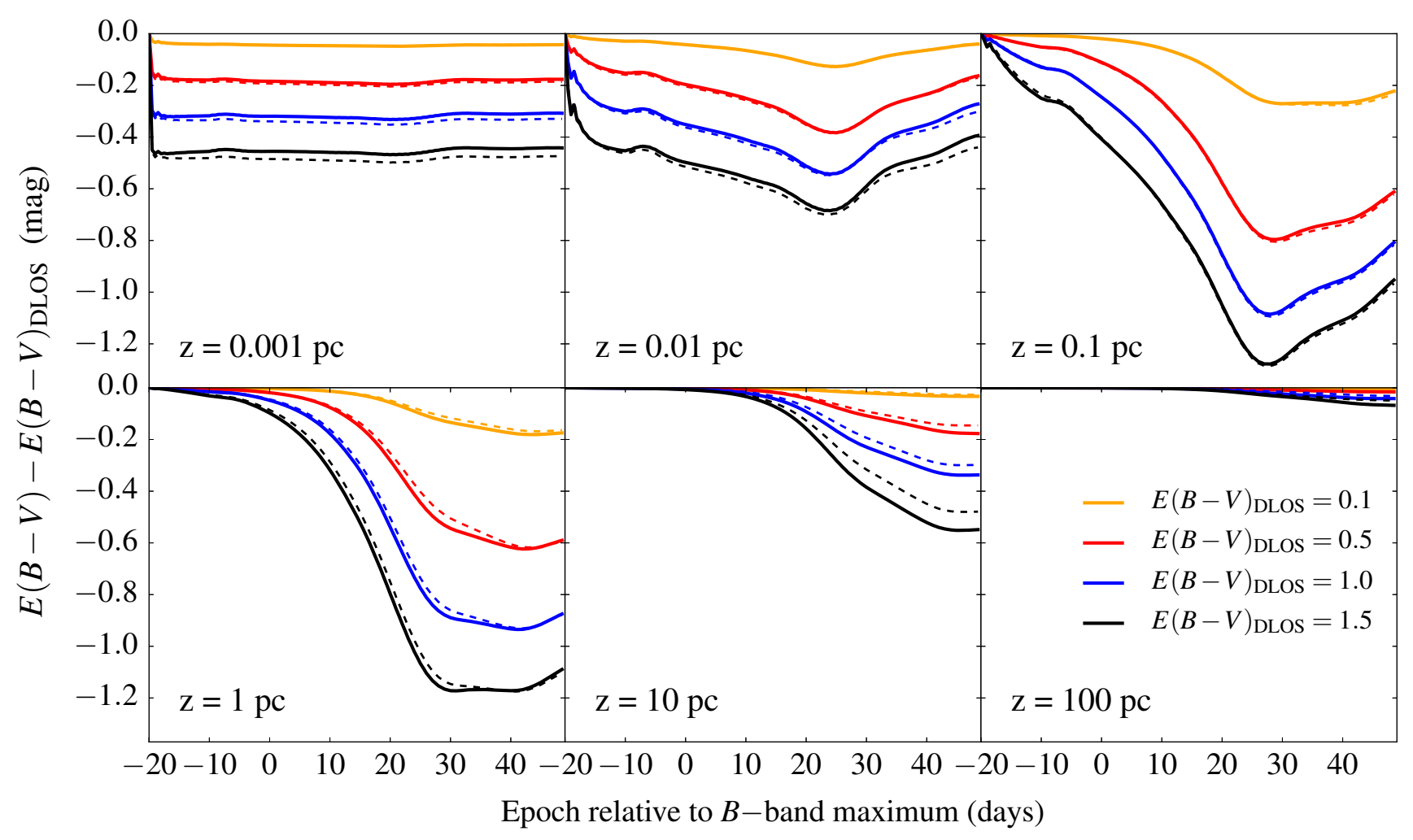

Figure 3. Temporal variation of the colour excess $E(B-V)$ for different $E(B-V)_{\mathrm{DLOS}}$ values and dust distances. Solid lines refer to LMC-type dust, while dashed lines to MW-type dust.

with limits inferred from the lack of infrared thermal emission in some normal SNe Ia $\left(\gtrsim 10^{18} \mathrm{~cm}\right.$, Johansson et al. $2013,2017)$. In addition, dust is unlikely to be present at $z \lesssim 10^{16} \mathrm{~cm}(\sim 0.003 \mathrm{pc})$ as at these distances it would be destroyed by the SN radiation (see e.g. discussion in Amanullah \& Goobar 2011).

Because of the broadening effect described above, the Hsiao template will be stretched by a convenient amount when comparing models to data in Section 3. In particular, for a given dust distance, we will choose the specific $\Delta M_{B, 15}^{\mathrm{em}}$ value required to match the $\mathrm{SN}$ observed decline rate $\Delta M_{B, 15}^{\text {obs }} \leqslant \Delta M_{B, 15}^{\text {em }}$ after interaction with dust.

\subsubsection{Time-variable colour excess}

Fig. 3 shows the temporal variation of the colour excess $E(B-V)$ for different dust locations, compositions and extinction values. Overall, the general behaviour of $E(B-V)$ with time is similar for all the models and for the scope of our study can be characterized by two main phases:

- In a first phase, the relative contribution of photons scattered into the DLOS is negligible and the observed flux dominated by non-interacting photons with $\Delta t=0$. Each $X$-band light curve is simply dimmed of a constant $A_{X}$ value, and thus $E(B-V)$ is constant with time.

- In a second phase, the relative contribution of photons scattered into the DLOS and reaching the observer with some time delay $\Delta t>0$ is significant. Given that scattered photons reach the observer at different times, the extinction $A_{X}$ in each $X$-band is no longer constant with time. In addition, the wavelength-dependence of the scattering and absorption properties causes different time-evolution in different bands, hence $E(B-V)$ is also time-dependent.

Similarly to what is found for $\Delta M_{B, 15}^{\mathrm{obs}}$ (see Section 2.2.1), the evolution of $E(B-V)$ is weakly dependent on the dust composition, with LMC- and MW-type dust predicting similar curves. As expected, we notice that the overall change in $E(B-V)$ during the second phase is larger for higher values of $E(B-V)$ DLOS. In addition, the transition between the two phases occurs at different epochs depending on the dust location. For very small distances $(z \sim 0.001 \mathrm{pc})$, all scattered photons arrive at the observer with time delays $\Delta t \lesssim 1 \mathrm{~d}$ and thus $E(B-V)$ starts departing from $E(B-V)_{\text {DLOS }}$ on these short time-scales. As the dust distance increases, the time delays for scattered photons becomes larger and thus $E(B-V)$ starts deviating from $E(B-V)_{\text {DLOS }}$ on increasingly longer timescales. In particular, $E(B-V)$ starts to deviate from $E(B-V)_{\text {DLOs }}$ already before $B$-band maximum for dust located at $z \lesssim 1 \mathrm{pc}$, while only post-peak for dust located farther away.

An important aspect shown by Fig. 3 is that the time evolution of $E(B-V)$ is significantly different for dust with different extinctions $E(B-V)_{\text {DLOS }}$ and different locations $z$. Estimating how the $E(B-V)$ for reddened SNe Ia changes with time can thus place strong constraints on the dust location. In the next Section, we will use this model prediction to infer dust locations towards reddened SNe Ia. 


\section{COMPARISON TO OBSERVATIONS}

In this section, we compare the $E(B-V)$ evolution predicted by our models with that we calculate for historical highly-reddened SNe Ia, with the goal of estimating dust distances in these events. In particular, we test our technique by focusing on two well-studied SNe Ia for which dust distances have been estimated from light echo detections: SN 2006X (Section 3.1) and SN 2014J (Section 3.2).

Following Amanullah et al. (2014), $E(B-V)$ values for the two $\mathrm{SNe}$ are calculated by comparing their observed colours to those of SN 2011fe, a normal "plain vanilla" SN Ia (Wheeler 2012) we take as representative of a pristine, unreddened $\mathrm{SN} \mathrm{Ia}^{3}$ due to its very-low extinction (Patat et al. 2013). The errors on the estimated $E(B-V)$ values are mostly dominated by the assumed conservative uncertainties on the intrinsic colour scatter in SNe Ia $(0.15 \mathrm{mag}$ in the $B$ and $V$ bands, see Amanullah et al. 2014). In our study, we choose to average photometric points of each SN in time-windows of 10 (SN 2006X) and 5 (SN 2014J) days in order to predict $E(B-V)$ values with uncertainties smaller than 0.05 mag.

\subsection{SN 2006X}

SN 2006X was discovered by Suzuki \& Migliardi (2006) in the M100 galaxy and found to suffer heavy host extinction. In particular, using photometric data, Wang et al. (2008a) derived an averaged colour excess for SN 2006X of $E(B-V)=1.42 \pm 0.04 \mathrm{mag}$. Here we use multi-band photometric data from tables 3 and 4 of Wang et al. (2008a) to investigate the evolution of $E(B-V)$ with time. Five phase intervals are chosen, and the $E(B-V)$ values extracted in each interval are reported in Table 1 . We notice that $E(B-V)$ is roughly constant and close to $1.5 \mathrm{mag}$ from about -4 to $+23 \mathrm{~d}$ relative to maximum, while we observe a drop to $E(B-V) \sim 1.3$ mag around +35 d.

Colour excess values thus estimated are shown in the left panel of Fig. 4, together with curves predicted by a series of LMC-type dust models. These models refer to dust distances from 10 to $50 \mathrm{pc}$, with a step size of $1 \mathrm{pc}$. To overcome our lack of knowledge about the true $E(B-V)_{\text {DLOS }}$ value, we use models with $E(B-V)_{\mathrm{DLOS}}=1.5 \mathrm{mag}$ but apply a shift of $+0.015 \mathrm{mag}$ so that modelled curves at early times are equal to the averaged value of the first two epochs of SN 2006X. As we can see, all the selected models predict a post-maximum drop in the $E(B-V)$ value compatible with that observed in SN 2006X. To select the most-probable distance, we perform a simple $\chi^{2}$ test for each modelled curve. As illustrated in the right panel of Fig. 4, we find a mostprobable distance of $z=27.5_{-4.9}^{+9.0} \mathrm{pc}$.

In the left panel of Fig. 5, we compare colour excess values for SN 2006X with models of MW-type dust located between 10 and $50 \mathrm{pc}$ (step size of $1 \mathrm{pc}$ ). Similarly to what is found for LMC-type dust, MW-type dust models also predict a post-maximum drop in $E(B-V)$. In this

3 We note that colours of SN 2011fe are remarkably similar to those of the Hsiao templates used in our simulations (see Section 2.2), and this is particularly true for the colours $(B-V)$ and epochs $(\gtrsim-10 \mathrm{~d}$ relative to peak) investigated in this work (see figs. 11 and 12 of Pereira et al. 2013).
Table 1. Average time and extracted $E(B-V)$ values for each phase interval of SN 2006X. Times and $E(B-V)$ values correspond to the white diamond points in the left panels of Figs. 4 and 5 .

\begin{tabular}{ccc}
\hline $\begin{array}{c}\text { Phase } \\
\left.\text { (days from } M_{B, 0}^{\text {obs }}\right)\end{array}$ & $\begin{array}{c}t_{\text {ave }} \\
\left(\text { days from } M_{B, 0}^{\text {obs }}\right)\end{array}$ & $\begin{array}{c}E(B-V) \\
(\mathrm{mag})\end{array}$ \\
\hline$[-10,0]$ & -3.6 & $1.501 \pm 0.043$ \\
{$[0,+10]$} & +2.8 & $1.530 \pm 0.043$ \\
{$[+10,+20]$} & +14.7 & $1.463 \pm 0.044$ \\
{$[+20,+30]$} & +22.6 & $1.466 \pm 0.046$ \\
{$[+30,+40]$} & +34.6 & $1.328 \pm 0.046$ \\
\hline
\end{tabular}

case, however, the most-probable distance (see right panel of Fig. 5) is lower and characterized by smaller uncertainties: $z=22.1_{-3.8}^{+6.0} \mathrm{pc}$.

The reason why different dust compositions lead to different distance values and uncertainties is explained in Fig. 6, where we show the relative contribution of scattered photons in the $B-$ and $V$-band as a function of dust distance $z$. The different changes in $E(B-V)$ between LMC - and MW-type dust are directly related to the different number of scattered photons contributing to the total flux. Due to the smaller albedo values of MW- compared to LMC-type dust (see table 1 of Goobar 2008), the scattering contribution and thus the changes in $E(B-V)$ for a given $z$ are smaller in the former model. The best match to the observed $E(B-V)$ values is therefore found at lower $z$ for MW- compared to LMC-type dust. In addition, Fig. 6 shows that the scattering contribution decreases with dust distance and that this variation is steeper at lower $z$. Moving to increasingly smaller values of $z$, the change in $E(B-V)$ is thus faster and the modelled curves of Figs. 4 and 5 less and less similar. As a consequence, the lower distance found in the MW-type dust model is also affected by smaller uncertainties.

Distance values derived above can be compared to estimates made for SN 2006X using different techniques. In particular, detections of light echoes in SN 2006X have been reported independently by Wang et al. (2008b) and Crotts \& Yourdon (2008) and used to constrain the dust distance under the single-scattering assumption. Based on Hubble Space Telescope (HST) data obtained $300 \mathrm{~d}$ post-maximum, Wang et al. (2008b) concluded that a thick dust layer should be located somewhere between $\sim 27$ and 170 pc away from the SN site. Combining these same measurements with HST data obtained 680 d post-maximum, Crotts \& Yourdon (2008) inferred a dust distance of $26.3 \pm 3.2 \mathrm{pc}$. The latter is in excellent agreement with the values obtained from our modelling. Specifically, their estimate is consistent with predictions from our LMC- and MW-type dust models within 0.6 and $0.2 \sigma$, respectively.

As discussed in Section 2.2.1, the SN templates used to calculate modelled curves were stretched so that the resulting light curve after interaction with dust has $\Delta M_{B, 15}^{\text {obs }}$ equal to the one observed for SN 2006X, $\Delta M_{B, 15}^{06 \mathrm{X}}=1.17 \pm 0.05 \mathrm{mag}$ (Wang et al. 2008a). As a by-product of our calculations, we can thus estimate the intrinsic decline rate of SN 2006X for the mostprobable distance values derived above. An intrinsic 

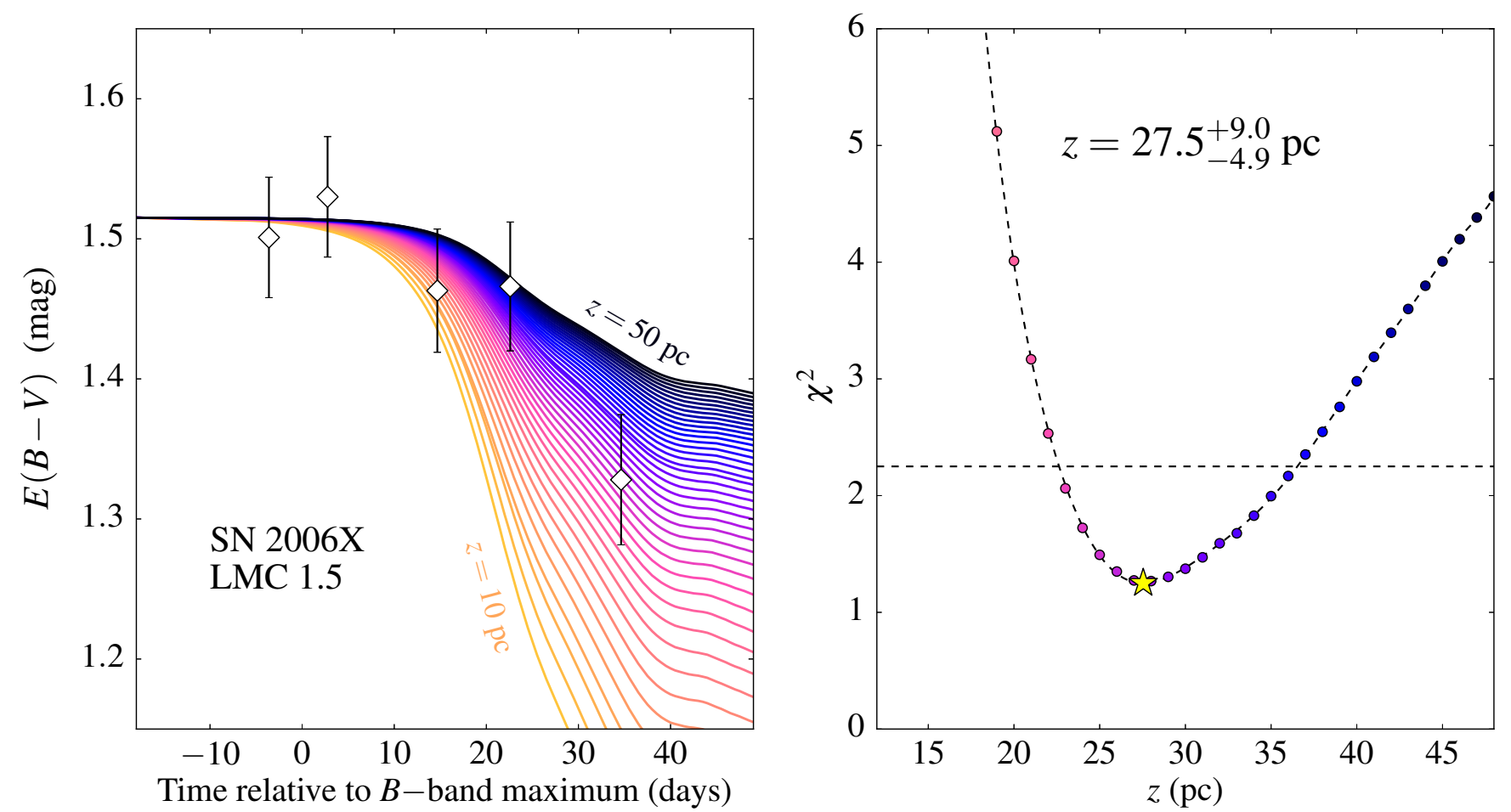

Figure 4. Left panel. $E(B-V)$ temporal evolution of SN 2006X (white diamonds) compared to model predictions for LMC-type dust with $E(B-V)_{\mathrm{DLOS}}=1.5$ mag. Modelled curves are calculated between 10 and $50 \mathrm{pc}$ (from orange to black) with a step size of 1 pc, and have been shifted by +0.015 mag to match the averaged value of the first two epochs of SN 2006X. Right panel. $\chi^{2}$ as a function of dust distances, where each point corresponds to a curve in the left panel. A 12th-order polynomial fitting (dashed curve) is performed and used to identify the most-probable distance (yellow star) and its uncertainties. The corresponding values are also reported in the panel.
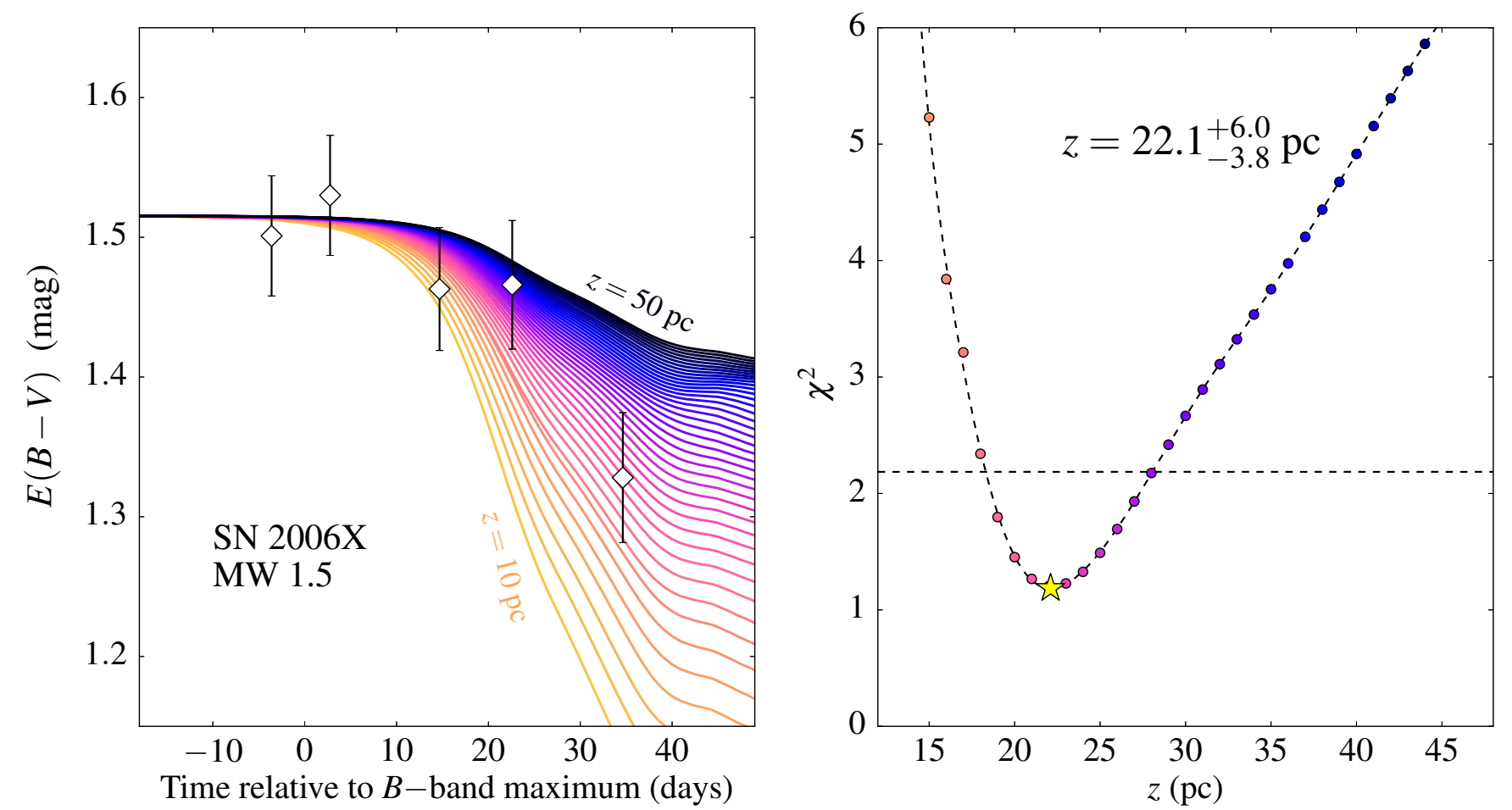

Figure 5. Same as Fig. 4 but for MW-type dust. 


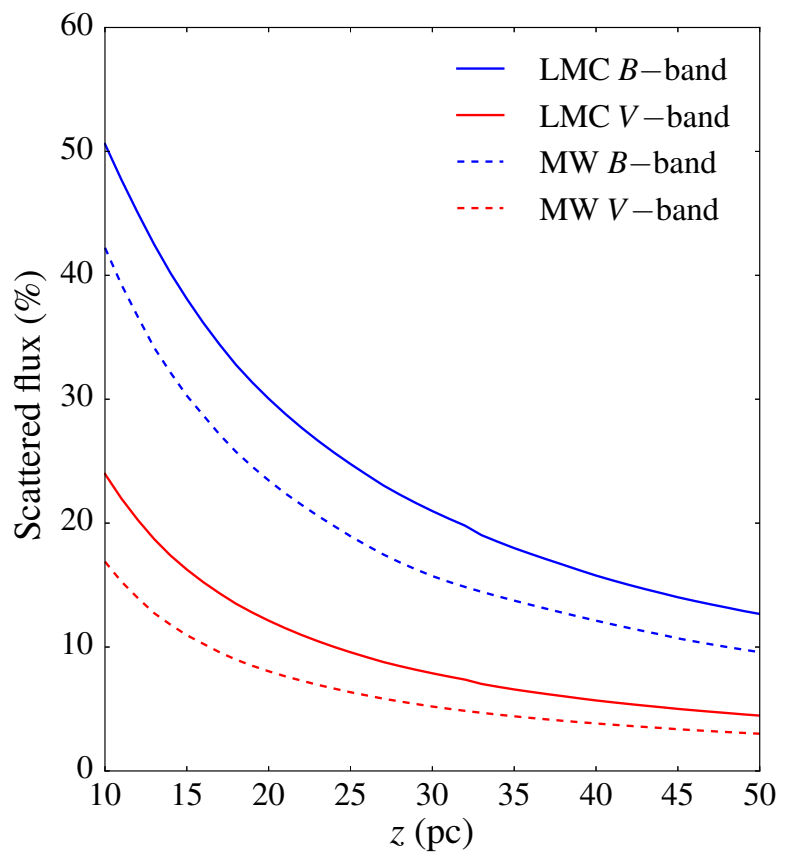

Figure 6. Relative contribution of scattering photons to the total flux in the $B-$ (blue lines) and $V$-band (red lines). Solid lines refer to LMC-type dust, while dashed lines to MW-type dust. Curves are calculated $35 \mathrm{~d}$ after $B$-band maximum, in correspondence to the last $E(B-V)$ value for SN 2006X (see Table 1).

decline rate of $\Delta M_{B, 15}^{\mathrm{em}}=1.20 \pm 0.01 \mathrm{mag}$ is required for both LMC-type dust at $z=27.5_{-4.9}^{+9.0} \mathrm{pc}$ and MW-type dust at $z=22.1_{-3.8}^{+6.0}$ pc. This decline rate is consistent with - although more precise than - the value derived by Wang et al. (2008a) using the empirical relation proposed by Phillips et al. (1999), $\Delta M_{B, 15}^{\mathrm{em}} \sim \Delta M_{B, 15}^{\mathrm{obs}}+0.1 \times E(B-V)=1.31 \pm 0.05 \mathrm{mag}$.

\section{$3.2 \quad$ SN 2014J}

SN 2014J was discovered in the nearby galaxy M82 and, owing to its proximity ( $D \sim 3.53 \mathrm{Mpc}$ ), has been the subject of several studies in the past few years. SN 2014J was found to suffer significant extinction from the host galaxy, with an averaged $E(B-V)$ value between $\sim 1.2$ and $1.4 \mathrm{mag}$ (Amanullah et al. 2014; Tsvetkov et al. 2014; Foley et al. 2014; Brown et al. 2015; Amanullah et al. 2015; Marion et al. 2015). Here we use multi-band photometry from Amanullah et al. (2015) to study the evolution of $E(B-V)$ with time. Six phase intervals are selected, and the $E(B-V)$ values for each interval are reported in Table 2. Unlike SN 2006X, SN 2014J does not show any clear evolution in the derived colour excess, with values extracted between -5 and $+25 \mathrm{~d}$ compatible with a constant $E(B-V) \sim 1.3$ mag. The lack of variability is consistent with the findings from Brown et al. (2015) while in conflict with the time-evolution suggested by Foley et al. (2014).

The left panel of Fig. 7 shows colour excess values estimated for SN 2014J, together with predicted curves from our LMC-type dust models. To encompass a larger distance range compared to that investigated for SN 2006X, here models are calculated between 25 and 75 pc (step size
Table 2. Same as Table 1 but for SN 2014J. Times and $E(B-V)$ values correspond to the white diamond points in the left panels of Figs. 7 and 8.

\begin{tabular}{ccc}
\hline $\begin{array}{c}\text { Phase } \\
\left(\text { days from } M_{B, 0}^{\text {obs }}\right)\end{array}$ & $\begin{array}{c}t_{\text {ave }} \\
\left(\text { days from } M_{B, 0}^{\text {obs }}\right)\end{array}$ & $\begin{array}{c}E(B-V) \\
(\mathrm{mag})\end{array}$ \\
\hline$[-5,0]$ & -2.6 & $1.337 \pm 0.027$ \\
{$[0,+5]$} & +1.1 & $1.313 \pm 0.027$ \\
{$[+5,+10]$} & +8.1 & $1.315 \pm 0.028$ \\
{$[+10,+15]$} & +17.6 & $1.314 \pm 0.046$ \\
{$[+15,+20]$} & +21.2 & $1.337 \pm 0.035$ \\
{$[+20,+25]$} & +29.0 & $1.290 \pm 0.047$ \\
\hline
\end{tabular}

of 5 pc) and between 100 and 500 pc (step size of 25 pc). We use models with $E(B-V)_{\text {DLOS }}=1.3$ mag but apply a shift of $+0.025 \mathrm{mag}$ to the extracted curves to match the averaged value of the first two epochs of SN 2014J. As shown in the right panel of Fig. 7, the most-probable dust distance is constrained to be $z \sim 121$ pc. Modelled curves at larger distances, however, tend to a constant $E(B-V)=1.325 \mathrm{mag}$ and are all consistent with SN 2014J at the epochs investigated. Hence, we infer a $1 \sigma$ lower limit on the distance and conclude that dust towards SN 2014J is located at $z \gtrsim 50 \mathrm{pc}$.

Fig. 8 presents a comparison between $E(B-V)$ values estimated for SN 2014J and those predicted by MW-type dust models. Predicted curves are calculated for the same distance range of LMC-type models. For the same reason discussed in Section 3.1, distance values estimated for MW-type dust are smaller than those for LMC-type dust. Specifically, we find that models with dust at $z \gtrsim 38 \mathrm{pc}$ are consistent with data within $1 \sigma$ and infer a most-probable distance of $z \sim 102$ pc.

Light echo detections towards SN 2014J were reported by Crotts (2015) and Yang et al. (2017). Using HST imaging data taken 216 d after $B$-band maximum, Crotts (2015) reported the detection of a light echo corresponding to dust at $\sim 330$ pc and of a possible inner one at $\sim 80$ pc. Combining these data with additional HST observations at $+277,+365$ and +416 d, Yang et al. (2017) confirmed the presence of the outer echo for which, however, they inferred a smaller and more precise distance of $228 \pm 7$ pc. In addition, they detected a diffuse light echo indicative of dust located between $\sim 100$ and 500 pc away from the SN. Distance values inferred by both studies are compatible with our lower limits for LMC- and MW-type dust models.

When calculating modelled $E(B-V)$ curves above, $\mathrm{SN}$ templates were stretched so that the observed decline rate after interaction with dust matches that inferred for SN 2014J. In particular, following Galbany et al. (2016) we averaged four different estimates in the literature (Tsvetkov et al. 2014; Ashall et al. 2014; Kawabata et al. 2014; Marion et al. 2015) and use $\Delta M_{B, 15}^{14 \mathrm{~J}}=1.06 \pm 0.06 \mathrm{mag}$. For both LMC - and MW-type dust, lower limits derived above imply an intrinsic decline rate $\Delta M_{B, 15}^{\mathrm{em}}=1.07 \pm 0.01 \mathrm{mag}$. In contrast, assuming $E(B-V)=1.3 \pm 0.1 \mathrm{mag}$, the empirical relation from Phillips et al. (1999, see Section 3.1) would give an intrinsic decline rate of $\Delta M_{B, 15}^{\mathrm{em}}=1.19 \pm 0.06 \mathrm{mag}$. The two different values are consistent with each others, although our estimate is more precise and not relying on empirical observations. 

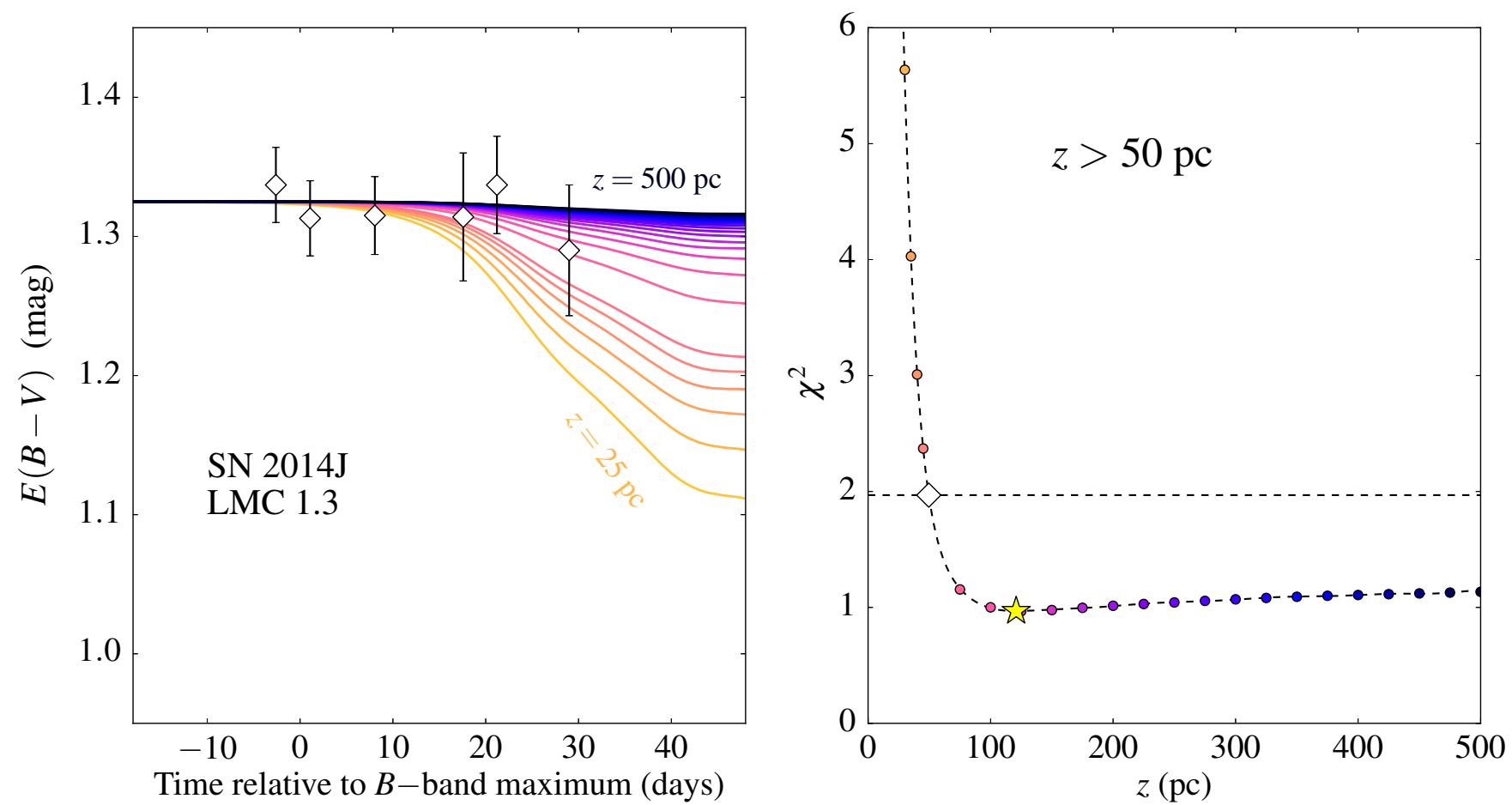

Figure 7. Left panel. $E(B-V)$ temporal evolution of SN 2014J (white diamonds) compared to model predictions for LMC-type dust with $E(B-V)_{\mathrm{DLOS}}=1.3 \mathrm{mag}$. Models are calculated between 25 and $75 \mathrm{pc}$ (step size of $5 \mathrm{pc}$ ) and between 100 and $500 \mathrm{pc}$ (step size of $25 \mathrm{pc}$ ). Model curves have been shifted by $+0.025 \mathrm{mag}$ to match the averaged value of the first two epochs of SN 2014J. Right panel. $\chi^{2}$ as a function of dust distances, where each point corresponds to a curve in the left panel. A 14th-order polynomial fitting (dashed curve) is performed and used to constraint the most-probable value (yellow star) and a lower limit (white diamond) for the dust distance. The latter value is also reported in the panel.

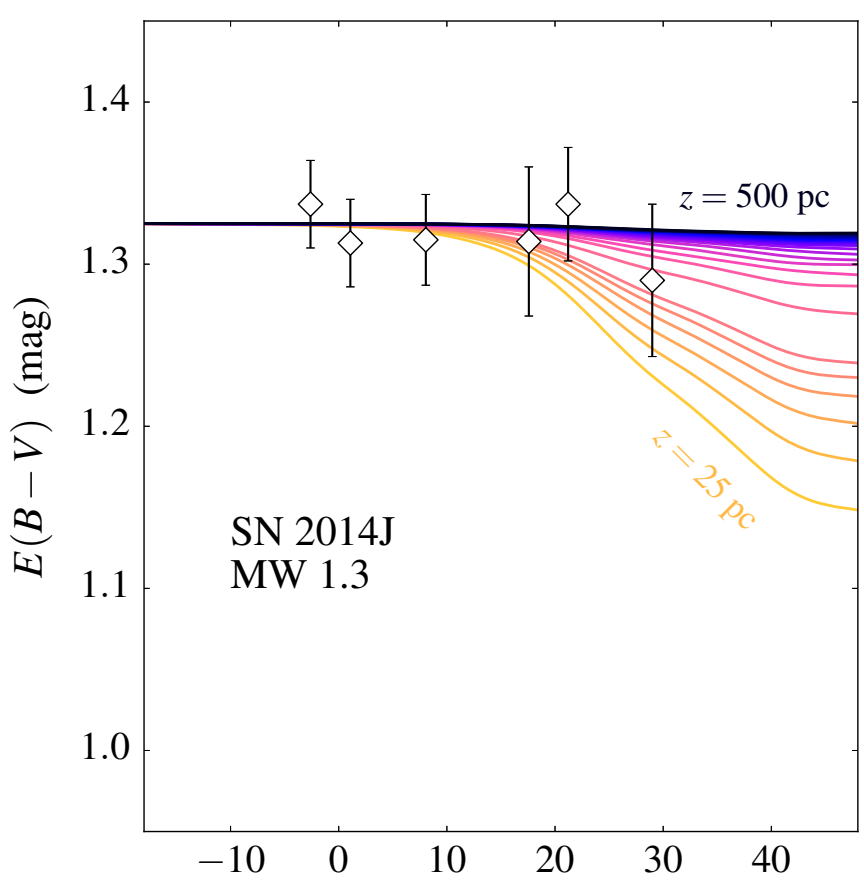

Time relative to $B$-band maximum (days)

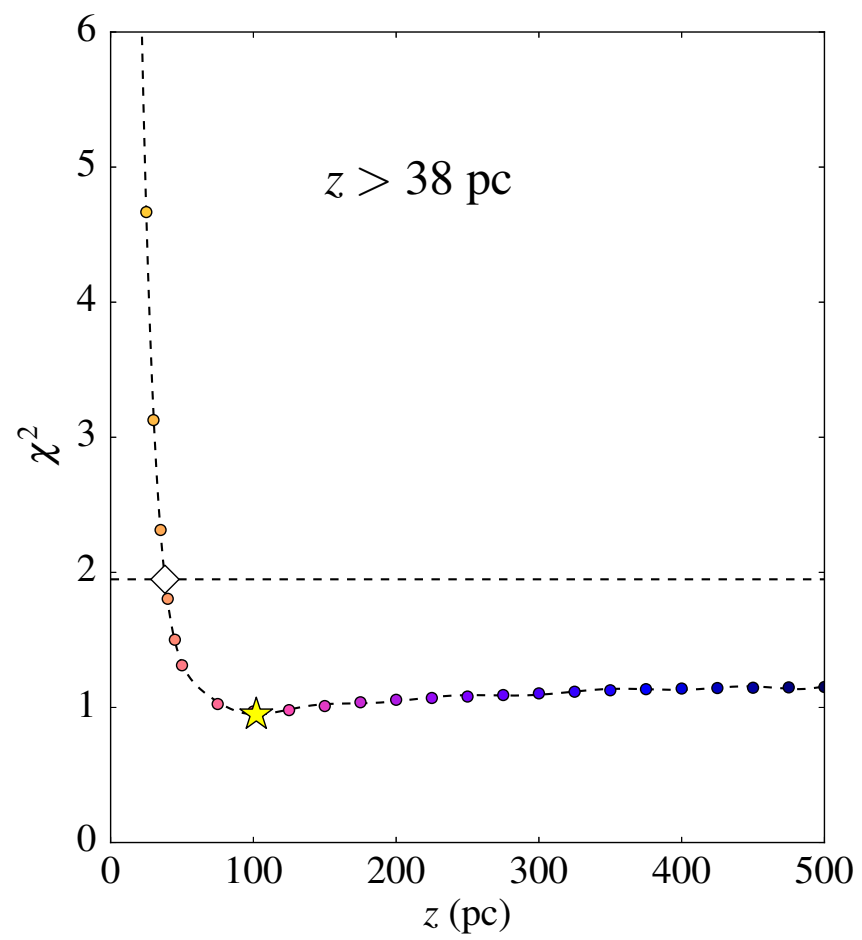

Figure 8. Same as Fig. 7 but for MW-type dust. 


\section{DISCUSSION}

In Section 3 we compared the $E(B-V)$ temporal evolution predicted by our LMC- and MW-type dust models with that inferred for two highly-extinguished SNe Ia, SN 2006X and SN 2014J. This comparison allowed us to place constraints on the dust location towards these SNe Ia. For SN 2006X, we obtain distance values of $z=27.5_{-4.9}^{+9.0} \mathrm{pc}$ and of $z=22.1_{-3.8}^{+6.0} \mathrm{pc}$ for LMC- and MW-type dust, respectively, in good agreement with previous estimates from light echo detections (26.3 $\pm 3.2 \mathrm{pc}$, Crotts \& Yourdon 2008). For SN 2014J, instead, our models suggest that dust is located at distances larger than $\sim 50$ and $38 \mathrm{pc}$ for LMC- and MW-type dust, respectively, again in good agreement with light echo measurements (Crotts 2015; Yang et al. 2017).

As already discussed in Section 2.2.1, a constant $E(B-V)$ in the time window covered by the observations (from around maximum to about $30 \mathrm{~d}$ after) is not only consistent with fairly distant but also with very nearby dust. Therefore, the $E(B-V)$ time evolution derived for SN 2014J could also be consistent with dust with $E(B-V)_{\mathrm{DLOS}} \sim 1.7 \mathrm{mag}$ and located at radii smaller than $\sim 10^{16} \mathrm{~cm}(\sim 0.003 \mathrm{pc}$, see Fig. 3$)$. Although we can not rule out this possibility, we find it rather unlikely since dust at these small distances should be either destroyed by the SN radiation (Amanullah \& Goobar 2011) or produce an infrared thermal emission that is not seen in SN 2014J and other normal SNe Ia (Johansson et al. 2013, 2017). We thus consider the very-distant dust case more plausible to explain the constant $E(B-V)$ behaviour observed in SN 2014J.

The dust region in our simulations is modelled as a single cloud with $E(B-V)_{\mathrm{DLOS}}$ (see Section 2.1). In the case of multiple clouds with the same combined $E(B-V)_{\mathrm{DLOS}}$, the results of this study would be unaffected. We note, however, that distance values estimated here would now be taken as representative of the outermost cloud (i.e. the more distant from the $\mathrm{SN}$ ).

Our findings suggest that, at least for the two SNe investigated, dust is likely to be located at IS-scale distances ( 10-100 pc). In the following, we briefly discuss how dust and gas at these scales might be compatible with other peculiar properties displayed by SNe Ia suffering dust extinction.

\subsection{Peculiar extinction and polarization}

As discussed in Section 1, highly-reddened SNe Ia are characterized by other peculiar properties. First, they are affected by unusually steep extinction law, with inferred $R_{\mathrm{V}}$ values significantly smaller than those typically observed for extinguished Galactic stars $\left(R_{\mathrm{V}} \sim 3.1\right)$. Secondly, they display a polarization curve peaking at shorter wavelengths than those typically seen for extinguished stars in our Galaxy $(\sim 0.55 \mu \mathrm{m})$.

To account for both these observational evidences, recent modelling efforts appear to favour an IS over a CS dust origin, provided that the dust grain sizes are significantly smaller than those found in the Galaxy (see e.g. Gao et al. 2015; Nozawa 2016; Hoang 2017). The scenario recently proposed by Hoang (2017) holds promises for explaining why the environment around some SNe Ia seems to prefer smaller dust grains. In this picture, a shift to smaller size distributions is believed to originate from cloud-cloud collisions induced by the SN radiation pressure. In particular, assuming that a number of different clouds are present along the DLOS, the more nearby clouds are first accelerated by the SN radiation pressure and later collides with more distant clouds. Cloud-cloud interactions are effective in destroying big grains, leading to the enhancement of grains smaller than $\sim 0.1 \mu \mathrm{m}$ and to the overall size distribution biased to smaller values.

The alignment of the polarization angle with the IS magnetic field of the host galaxy has been used as an additional strong argument favouring IS over CS dust scenarios (Patat et al. 2015; Zelaya et al. 2017). By modelling extinction and polarization data of the four SNe Ia studied by Patat et al. (2015), Hoang (2017) finds that the observed alignment is compatible with that predicted by the radiative torque (RAT) mechanism (see Lazarian et al. 2015 for a recent review). In particular, the alignment observed in three SNe Ia (SN 1986G, SN 2006X and SN 2014J) is consistent with that of our Galaxy - where big grains are more efficiently aligned than small grains - and ascribed to RAT induced by IS radiation fields. In contrast, modelling of SN 2008fp requires small grains to be aligned as efficiently as big grains. In the scenario described above, Hoang (2017) interprets this peculiar alignment as a consequence of RAT induced by the SN radiation itself, a picture that is found to work best for dust located at distances between $\sim 1$ and 20 pc.

Here we note that calculations presented in this work have been performed using MW- and LMC-type dust. With these compositions, dust clouds located at distances inferred from Section $3(\gtrsim 20 \mathrm{pc}$ ) are not able to reproduce the low $R_{\mathrm{V}}$ values observed in SNe Ia. Using a more appropriate grain size distribution as the one predicted by Hoang (2017) would likely lead to more compatible $R_{\mathrm{V}}$ values at these distance scales. The different dust composition would also lead to different $E(B-V)$ evolution and it thus might affect the distance estimates here derived. From the comparison in Fig. 3, however, we expect a different dust composition to only marginally affect the $E(B-V)$ evolution and hence the estimated dust location.

To summarize, the grain size distribution and alignment function calculated by Hoang (2017) are indicative of dust located at IS-scales ( $\gtrsim 1 \mathrm{pc}$ ), in good agreement with distances estimated in this work (Section 3) and with those inferred from light echo detections towards some SNe Ia.

\subsection{Variable blue-shifted absorptions}

For the two SNe Ia investigated in this study, variable, blueshifted components have been detected in the $\mathrm{NaI} \mathrm{D}$ and K I absorption profiles. Variable sodium was observed in SN 2006X (Patat et al. 2007), while SN 2014J showed variability in the potassium profile but not in the sodium one (Goobar et al. 2014; Foley et al. 2014; Ritchey et al. 2015; Graham et al. 2015; Jack et al. 2015; Maeda et al. 2016). These variations were interpreted as arising from outflowing CS gas where sodium and potassium are subject to photoionization and later recombination effects (Patat et al. 2007; Graham et al. 2015, but see Maeda et al. 2016 for an alternative interpretation in the IS scenario). To explain the observed variabilities, the gas was inferred to be at distances $(0.1-1.0) \times 10^{17} \mathrm{~cm}(\sim 0.003-0.03 \mathrm{pc})$ in SN 2006X 
(Patat et al. 2007), while $(0.6-1.6) \times 10^{19} \mathrm{~cm}(\sim 2-5 \mathrm{pc})$ in SN 2014J (Graham et al. 2015).

In order for these components to originate within IS clouds (i.e. at distances where dust in SN 2006X and SN 2014J appears to be located), one would need to invoke some geometric effect. For instance, the varying absorption features might stem from IS clouds whose covering factor changes with time due to the rapid expansion of the underlying SN photosphere. This possibility was in part ruled out by Patat et al. (2010), which found that the variations in the Na I D equivalent width $(\lesssim 40 \mathrm{~mA})$ induced by a fractal patchy IS medium are much smaller than those observed in some SNe Ia (e.g. $300 \mathrm{~mA}$ for SN 2006X). Large variations as those seen in SN 2006X, however, could still be consistent with geometric effects if the IS medium is highly nonhomogeneous. For instance, Chugai (2008) suggested that the strongest variability observed in the $\mathrm{NaI} \mathrm{D}$ profile of SN 2006X ("D" component in Patat et al. 2007) can be explained if the SN is first seen through the hole of a cluster of clouds while later partially occulted by the cluster. Using our Monte Carlo code, we verified that this particular distribution of the IS clouds can indeed reproduce the variability observed in SN 2006X.

A common argument against geometric effects in IS clouds relies on the fact that the observed blue-shifted components of NaI D, Ca II H\&K and K I do not vary simultaneously (Patat et al. 2007). For instance, the blue-shifted components observed in the Ca II H\&K profile of SN 2006X did not show any time evolution as was the case for the $\mathrm{Na}$ I D. In the CS scenario, instead, the lack of variability of calcium has been explained in terms of the higher ionization potential of calcium compared to sodium (Patat et al. 2007). This argument has been challenged by Chugai (2008) who pointed out that this is also expected in the IS scenario provided that the variable sodium components arise from clouds where calcium is depleted on dust grains.

Another issue for the IS scenario is to explain why the variable components are preferentially seen blue-shifted. A possible answer is found in the scenario proposed by Hoang (2017), where small grain sizes inferred from extinction and polarization analysis are due to cloud-cloud collisions induce by $\mathrm{SN}$ radiation (see Section 4.1). In this picture, the SN radiation pressure accelerates the more nearby clouds to move towards the observer and to later collide with the more distant clouds. Absorbing gas in these moving clouds would thus be detected via blue-shifted components. In addition, we note that the scenario of Hoang (2017) can also account for the observational preponderance of variable $\mathrm{Na}$ I D and $\mathrm{K}$ I features in SNe Ia with large dust extinction (Patat et al. 2007; Blondin et al. 2009; Phillips et al. 2013; Graham et al. 2015). In fact, cloud-cloud collisions would not only destroy big grains to smaller sizes (see Section 4.1) but also lead to an enhancement in the gaseous phase of atoms such as sodium, calcium and potassium. Finally, this scenario might also explain why the SNe Ia with unusually high sodium absorption in the sample studied by Phillips et al. (2013) display blue-shifted Na I D absorptions.

In summary, although variable blue-shifted components have been historically explained by CS gas around some SNe Ia, growing evidences suggest that these can also be consistent with geometric effects arising at IS-scale distances.

\section{CONCLUSIONS}

We have presented a new approach to calculate the location of dust in reddened SNe Ia. This technique exploits the fact that dust regions at different locations produce distinct behaviours on the observed SN light curve (Amanullah \& Goobar 2011). Using Monte Carlo calculations, we have shown that the detailed evolution of the colour excess $E(B-V)$ provides strong constraints on the dust distance from the SN. Although this approach is complementary to distance estimates from light echo measurements, unlike the latter it does not require to obtain late-time HST imaging but only photometric data at relatively earlier epochs. Moreover, the use of Monte Carlo methods naturally incorporates the effect of multiple scattering on dust particles which is not taken into account when estimating distances from light echo measurements.

We applied our approach to two well-studied SNe Ia suffering large extinction: SN 2006X and SN 2014J. In the case of SN 2006X, our models predict SN-dust distances of $z=27.5_{-4.9}^{+9.0}$ and $z=22.1_{-3.8}^{+6.0} \mathrm{pc}$ depending on whether LMC- or MW-type dust is used. These values are in excellent agreement with predictions from light echo detections (26.3 $\pm 3.2 \mathrm{pc}$, Crotts \& Yourdon 2008). In the case of SN 2014J, $E(B-V)$ is found to be constant in the time window investigated, allowing us to place only lower limits on the dust distance. Specifically, dust is constrained to be located at distances larger than $\sim 50$ and $38 \mathrm{pc}$ for LMC- and MW-type dust, respectively, in good agreement with light echo suggestions (Crotts 2015; Yang et al. 2017).

Our study indicates that for the two SNe Ia investigated dust is likely to be located at IS-scale distances. This is in conflict with findings from Cikota et al. (2017), which support multiple scattering in a local environment for these SNe, but compatible with the non-detection of infrared thermal emission in SN 2014J (Johansson et al. 2017). We have discussed how our results could be consistent with other peculiar properties displayed by some highly-reddened SNe Ia. First, their peculiar extinction and polarization properties could be ascribed to small IS grains produced by cloud-cloud collisions induced by the SN radiation pressure, as proposed by Hoang (2017). Secondly, the variable, blue-shifted components observed in Na I D and K I features of some highlyreddened SNe Ia (including SN 2006X and SN 2014J) could be a consequence of the SN photosphere expanding in a nonhomogeneous IS medium (Chugai 2008). In particular, we confirmed this possibility with our Monte Carlo code by exploring a similar geometry to the one proposed by Chugai (2008). Finally, the cloud-cloud collision scenario proposed by Hoang (2017) might explain why SNe Ia with unusually strong NaI D absorption are characterized by blueshifted Na I D components (Phillips et al. 2013) and why variabilities in the latter are preferentially seen in SNe Ia suffering large extinction.

While in this study we focused on the evolution of $E(B-V)$, in the future we will investigate the effect of dust on the extinction in different bands $A_{X}$ and thus on the colour excess $E(X-V)$. Combining information from different wavelengths will help provide even more stringent constraints on the dust location. Also, since scattering and absorption properties have not been computed for the grain distribution predicted by Hoang (2017), in this study we 
have restricted to using LMC - and MW-type dust composition. As a result, our models predict $R_{\mathrm{V}}$ values consistent with those observed in the Galaxy (and thus inconsistent with $\mathrm{SNe} \mathrm{Ia}$ ) at the pc-scale distances inferred from the $E(B-V)$ evolution. In the future, we plan to perform Monte Carlo calculations using dust properties inferred from Hoang (2017). When placing dust at IS distances, we expect these calculations to give $R_{\mathrm{V}}$ values consistent with those seen in SNe Ia, while only marginally affecting the $E(B-V)$ time evolution and thus distance estimates derived in this study.

Although in this work we focused on presenting and testing our technique on two-well known SNe Ia, in the future we plan to apply the same approach to a larger sample of highly-reddened SNe Ia. In particular, the first year of the upcoming wide-field ground-based Zwicky Transient Facility (ZTF, Kulkarni 2016) survey will provide us with roughly 100 (40) SNe Ia with host $E(B-V) \geqslant 0.4(0.5)$ mag, 70 (25) of which will be discovered before maximum light. This will allow us to estimate colour excess evolutions and thus dust distance locations for a statistically-significant sample, and possibly help solve the CS vs IS long-standing debate.

\section{ACKNOWLEDGEMENTS}

The authors are thankful to the anonymous reviewer for his/her valuable comments. The authors acknowledge support from the Swedish Research Council (Vetenskapsrådet) and the Swedish National Space Board.

\section{REFERENCES}

Amanullah R., Goobar A., 2011, ApJ, 735, 20

Amanullah R., Goobar A., Johansson J., Banerjee D. P. K., Venkataraman V., Joshi V., Ashok N. M., Cao Y., Kasliwal M. M., Kulkarni S. R., Nugent P. E., Petrushevska T., Stanishev V., 2014, ApJL, 788, L21

Amanullah R., Johansson J., Goobar A., Ferretti R., Papadogiannakis S., Petrushevska T., Brown P. J., Cao Y., Contreras C., Dahle H., et al., 2015, MNRAS, 453, 3300

Ashall C., Mazzali P., Bersier D., Hachinger S., Phillips M., Percival S., James P., Maguire K., 2014, MNRAS, 445, 4427

Astier P., Guy J., Regnault N., Pain R., Aubourg E., Balam D., Basa S., Carlberg R. G., Fabbro S., Fouchez D., et al., 2006, A\&A, 447, 31

Blondin S., Prieto J. L., Patat F., Challis P., Hicken M., Kirshner R. P., Matheson T., Modjaz M., 2009, ApJ, 693, 207

Brown P. J., Smitka M. T., Wang L., Breeveld A., de Pasquale M., Hartmann D. H., Krisciunas K., Kuin N. P., Milne P. A., Page M., Siegel M., 2015, ApJ, 805, 74

Burns C. R., Stritzinger M., Phillips M. M., Hsiao E. Y., Contreras C., Persson S. E., Folatelli G., Boldt L., Campillay A., Castellón S., Freedman W. L., Madore B. F., Morrell N., Salgado F., Suntzeff N. B., 2014, ApJ, 789, 32

Cappellaro E., Patat F., Mazzali P. A., Benetti S., Danziger J. I., Pastorello A., Rizzi L., Salvo M., Turatto M., 2001, ApJL, 549, L215

Chugai N. N., 2008, Astronomy Letters, 34, 389
Cikota A., Patat F., Cikota S., Spyromilio J., Rau G., 2017, preprint (arXiv:1708.02300)

Crotts A. P. S., 2015, ApJL, 804, L37

Crotts A. P. S., Yourdon D., 2008, ApJ, 689, 1186

Draine B. T., 2003, ApJ, 598, 1017

Drozdov D., Leising M. D., Milne P. A., Pearcy J., Riess A. G., Macri L. M., Bryngelson G. L., Garnavich P. M., 2015, ApJ, 805, 71

Elias-Rosa N., Benetti S., Cappellaro E., Turatto M., Mazzali P. A., Patat F., Meikle W. P. S., Stehle M., Pastorello A., Pignata G., et al., 2006, MNRAS, 369, 1880

Elias-Rosa N., Benetti S., Turatto M., Cappellaro E., Valenti S., Arkharov A. A., Beckman J. E., di Paola A., Dolci M., Filippenko A. V., et al., 2008, MNRAS, 384, 107

Ferretti R., Amanullah R., Goobar A., Johansson J., Vreeswijk P. M., Butler R. P., Cao Y., Cenko S. B., Doran G., Filippenko A. V., et al., 2016, A\&A, 592, A40

Fitzpatrick E. L., Massa D., 2007, ApJ, 663, 320

Folatelli G., Phillips M. M., Burns C. R., Contreras C., Hamuy M., Freedman W. L., Persson S. E., Stritzinger M., Suntzeff N. B., Krisciunas K., et al., 2010, AJ, 139, 120

Foley R. J., Fox O. D., McCully C., Phillips M. M., Sand D. J., Zheng W., Challis P., Filippenko A. V., Folatelli G., Hillebrandt W., et al., 2014, MNRAS, 443, 2887

Foley R. J., Kasen D., 2011, ApJ, 729, 55

Foley R. J., Simon J. D., Burns C. R., Gal-Yam A., Hamuy M., Kirshner R. P., Morrell N. I., Phillips M. M., Shields G. A., Sternberg A., 2012, ApJ, 752, 101

Galbany L., Moreno-Raya M. E., Ruiz-Lapuente P., González Hernández J. I., Méndez J., Vallely P., Baron E., Domínguez I., Hamuy M., López-Sánchez A. R., et al., 2016, MNRAS, 457, 525

Gao J., Jiang B. W., Li A., Li J., Wang X., 2015, ApJL, 807, L26

Goobar A., 2008, ApJL, 686, L103

Goobar A., Johansson J., Amanullah R., Cao Y., Perley D. A., Kasliwal M. M., Ferretti R., Nugent P. E., Harris C., Gal-Yam A., et al., 2014, ApJL, 784, L12

Graham M. L., Valenti S., Fulton B. J., Weiss L. M., Shen K. J., Kelly P. L., Zheng W., Filippenko A. V., Marcy G. W., Howell D. A., et al., 2015, ApJ, 801, 136

Henyey L. G., Greenstein J. L., 1941, ApJ, 93, 70

Hillebrandt W., Kromer M., Röpke F. K., Ruiter A. J., 2013, Frontiers of Physics, 8, 116

Hoang T., 2017, ApJ, 836, 13

Hsiao E. Y., Conley A., Howell D. A., Sullivan M., Pritchet C. J., Carlberg R. G., Nugent P. E., Phillips M. M., 2007, ApJ, 663, 1187

Jack D., Mittag M., Schröder K.-P., Schmitt J. H. M. M., Hempelmann A., González-Pérez J. N., Trinidad M. A., Rauw G., Cabrera Sixto J. M., 2015, MNRAS, 451, 4104 Johansson J., Amanullah R., Goobar A., 2013, MNRAS, 431, L43

Johansson J., Goobar A., Kasliwal M. M., Helou G., Masci F., Tinyanont S., Jencson J., Cao Y., Fox O. D., Kromer M., Amanullah R., Banerjee D. P. K., Joshi V., Jerkstrand A., Kankare E., Prince T. A., 2017, MNRAS, 466, 3442

Kawabata K. S., Akitaya H., Yamanaka M., Itoh R., Maeda K., Moritani Y., Ui T., Kawabata M., Mori K., Nogami D., et al., 2014, ApJL, 795, L4 
Kowalski M., Rubin D., Aldering G., Agostinho R. J., Amadon A., Amanullah R., Balland C., Barbary K., Blanc G., Challis P. J., et al., 2008, ApJ, 686, 749

Krisciunas K., Prieto J. L., Garnavich P. M., Riley J.-L. G., Rest A., Stubbs C., McMillan R., 2006, AJ, 131, 1639

Kulkarni S. R., 2016, in American Astronomical Society Meeting Abstracts Vol. 227 of American Astronomical Society Meeting Abstracts, The Zwicky Transient Facility. p. 314.01

Lazarian A., Andersson B.-G., Hoang T., 2015, Polarimetry of Stars and Planetary Systems, p. 81

Maeda K., Tajitsu A., Kawabata K. S., Foley R. J., Honda S., Moritani Y., Tanaka M., Hashimoto O., Ishigaki M., Simon J. D., et al., 2016, ApJ, 816, 57

Maguire K., Sullivan M., Patat F., Gal-Yam A., Hook I. M., Dhawan S., Howell D. A., Mazzali P., Nugent P. E., Pan Y.-C., et al., 2013, MNRAS, 436, 222

Marion G. H., Sand D. J., Hsiao E. Y., Banerjee D. P. K., Valenti S., Stritzinger M. D., Vinkó J., Joshi V., Venkataraman V., Ashok N. M., et al., 2015, ApJ, 798,39

Nobili S., Goobar A., 2008, A\&A, 487, 19

Nozawa T., 2016, Planetary and Space Science, 133, 36

Patat F., Benetti S., Cappellaro E., Turatto M., 2006, MNRAS, 369, 1949

Patat F., Chandra P., Chevalier R., Justham S., Podsiadlowski P., Wolf C., Gal-Yam A., Pasquini L., Crawford I. A., Mazzali P. A., et al., 2007, Science, 317, 924

Patat F., Cordiner M. A., Cox N. L. J., Anderson R. I., Harutyunyan A., Kotak R., Palaversa L., Stanishev V., Tomasella L., Benetti S., Goobar A., Pastorello A., Sollerman J., 2013, A\&A, 549, A62

Patat F., Cox N. L. J., Parrent J., Branch D., 2010, A\&A, 514, A78

Patat F., Taubenberger S., Cox N. L. J., Baade D., Clocchiatti A., Höflich P., Maund J. R., Reilly E., Spyromilio J., Wang L., Wheeler J. C., Zelaya P., 2015, A\&A, 577, A53

Pereira R., Thomas R. C., Aldering G., Antilogus P., Baltay C., Benitez-Herrera S., Bongard S., Buton C., Canto A., Cellier-Holzem F., et al., 2013, A\&A, 554, A27

Perlmutter S., Aldering G., Goldhaber G., Knop R. A., Nugent P., Castro P. G., Deustua S., Fabbro S., Goobar A., Groom D. E., et al., 1999, ApJ, 517, 565

Phillips M. M., Lira P., Suntzeff N. B., Schommer R. A., Hamuy M., Maza J., 1999, AJ, 118, 1766

Phillips M. M., Simon J. D., Morrell N., Burns C. R., Cox N. L. J., Foley R. J., Karakas A. I., Patat F., Sternberg A., Williams R. E., et al., 2013, ApJ, 779, 38

Quinn J. L., Garnavich P. M., Li W., Panagia N., Riess A., Schmidt B. P., Della Valle M., 2006, ApJ, 652, 512

Riess A. G., Filippenko A. V., Challis P., Clocchiatti A., Diercks A., Garnavich P. M., Gilliland R. L., Hogan C. J., Jha S., Kirshner R. P., et al., 1998, ApJ, 116, 1009

Ritchey A. M., Welty D. E., Dahlstrom J. A., York D. G., 2015, ApJ, 799, 197

Schmidt B. P., Kirshner R. P., Leibundgut B., Wells L. A., Porter A. C., Ruiz-Lapuente P., Challis P., Filippenko A. V., 1994, ApJL, 434, L19

Serkowski K., Mathewson D. S., Ford V. L., 1975, ApJ, 196, 261

Simon J. D., Gal-Yam A., Gnat O., Quimby R. M., Gane- shalingam M., Silverman J. M., Blondin S., Li W., Filippenko A. V., Wheeler J. C., et al., 2009, ApJ, 702, 1157

Sparks W. B., Macchetto F., Panagia N., Boffi F. R., Branch D., Hazen M. L., Della Valle M., 1999, ApJ, 523, 585

Sternberg A., Gal-Yam A., Simon J. D., Leonard D. C., Quimby R. M., Phillips M. M., Morrell N., Thompson I. B., Ivans I., Marshall et al., 2011, Science, 333, 856

Suzuki S., Migliardi M., 2006, IAU Circ., 8667

Tripp R., 1998, A\&A, 331, 815

Tsvetkov D. Y., Metlov V. G., Shugarov S. Y., Tarasova T. N., Pavlyuk N. N., 2014, Contributions of the Astronomical Observatory Skalnate Pleso, 44, 67

Wang L., 2005, ApJL, 635, L33

Wang X., Li W., Filippenko A. V., Krisciunas K., Suntzeff N. B., Li J., Zhang T., Deng J., Foley R. J., Ganeshalingam M., Li T., Lou Y., Qiu Y., Shang R., Silverman J. M., Zhang S., Zhang Y., 2008a, ApJ, 675, 626

Wang X., Li W., Filippenko A. V., Foley R. J., Smith N., Wang L., 2008b, ApJ, 677, 1060

Wang X., Filippenko A. V., Ganeshalingam M., Li W., Silverman J. M., Wang L., Chornock R., Foley R. J., Gates E. L., Macomber B., Serduke F. J. D., Steele T. N., Wong D. S., 2009, ApJL, 699, L139

Weingartner J. C., Draine B. T., 2001, ApJ, 548, 296

Wheeler J. C., 2012, ApJ, 758, 123

Whittet D. C. B., van Breda I. G., 1978, A\&A, 66, 57

Witt A. N., 1977, ApJS, 35, 1

Yang Y., Wang L., Baade D., Brown P. J., Cracraft M., Höflich P. A., Maund J., Patat F., Sparks W. B., Spyromilio J., Stevance H. F., Wang X., Wheeler J. C., 2017, ApJ, 834, 60

Zelaya P., Clocchiatti A., Baade D., Höflich P., Maund J., Patat F., Quinn J. R., Reilly E., Wang L., Wheeler J. C., Förster F., González-Gaitán S., 2017, ApJ, 836, 88 